\title{
Imaging of Postpartum Uterine Pathology: A Review
}

\section{Aslı Üçyiğit* and Jemma Johns}

King's College Hospital, NHS Foundation Trust, London, United Kingdom

\begin{abstract}
A number of various imaging modalities are currently available for the assessment of the postpartum uterus and have a significant role to play in the evaluation of symptomatic puerperal women. Although magnetic resonance imaging and computed tomography have become more readily obtainable in recent times, ultrasound scanning is often the imaging modality of choice for excluding retained placental tissue and other postpartum pathology. The correct application of postpartum imaging could enable more accurate identification of women requiring surgical intervention, with consequent reduction in patient morbidity, radiation exposure, clinical workload and cost. In this article the current understanding and application of imaging modalities during the puerperium, in particular ultrasound, is reviewed. Furthermore, the existing evidence investigating the physiological and pathological findings of the postpartum uterus and its contents is explored.
\end{abstract}

\section{Introduction}

Postpartum uterine pathology is a common cause of morbidity and rarely, mortality in women of reproductive age. It is therefore paramount that clinicians are able to select the most appropriate imaging modality in order to aid accurate diagnosis and subsequent treatment of the postpartum patient.

It cannot be denied that a thorough history from and physical examination of the postpartum patient will more often than not provide the diagnosis, however confirmation is usually obtained through imaging. Portable and departmental ultrasound scanning has become an increasingly utilised investigative tool in the diagnosis of puerperal complications. The safety profile and patient acceptability rates of both transabdominal and transvaginal ultrasound scanning often render it the first-line imaging modality of choice when postpartum pathology is suspected. In contrast to MRI ant CT scanning, the accessibility and relative low cost of ultrasound scanners have allowed clinicians other than radiologists to undertake diagnostic imaging examinations. As a real-time imaging method, it can not only be used as an adjunct to bimanual examinationbut also utilised in the guidance of therapeutic procedures, such as evacuation, aspiration and drainage procedures. Ultrasound also negates the need for intravenous contrast material (often required to produce optimal CT or MRI images) which may interrupt breastfeeding; such agents are known to be excreted in small quantities in human breast milk. The main disadvantage of ultrasound is that it is operator dependent, and when patient body habitus is increased or bowel gas is present, deeper structures are difficult to assess. In these scenarios secondary imaging with CT or MRI may be required for further evaluation of suspected pathology.

It must be emphasised however, that an understanding of the normal appearance of the postpartum uterus is a prerequisite for the accurate diagnosis and management of puerperal pathology, no matter which imaging modality is used.

\section{Common Indications for Postpartum Imaging}

There are a number of possible indications for pelvic imaging, most commonly suspected retained products of conception or pelvic sepsis, both of which often present with excessive or erratic bleeding.

\section{Publication History:}

Received: July 25, 2017

Accepted: December 04, 2017

Published: December 06, 2017

\section{Keywords:}

Puerperium, Radiological appearances of uterus.
Primary postpartum haemorrhage $(\mathrm{PPH})$ is traditionally defined as the loss of at least $500 \mathrm{ml}$ of blood from the lower genital tract within 24 hours of delivery,or any blood loss that results in maternal haemodynamic compromise [1-4]. Although the most common cause of primary PPH is uterine atony, care must be taken to exclude retained products of conception (placenta and membranes) or intrauterine blood clots as an additional or primary cause [4]. Clearly any offward radiological assessment (e.g. CT or MRI scanning) is rarely indicated in this emergency setting, yet bedside ultrasound scanning can be a useful diagnostic adjunct to clinical examination in this scenario. In more complex cases, where intrabdominal haemorrhage is suspected, CT has been proposed as a superior mode of imaging, permitting localisation of arterial bleeding sites and haematomas, along with vascular mapping, which is necessary should endovascular embolisation therapy be required [5].

The occurrence of increased or abnormal genital tract bleeding occurring between 24 hours and 12 weeks postpartumis defined as secondary $\mathrm{PPH}$, and is often a sign of underlying endometritis or retained products of conception $[4,6]$. Abnormal bleeding up to six weeks is the more commonly used definition of secondary PPH in the UK and in developed countries $2 \%$ of postnatal women are admitted to hospital with such symptoms [7]. Up to half of these women will undergo surgical evacuation in accordance with the guidance published by the Royal College of Obstetricians and Gynaecologists, who recommend surgical treatment when there is excessive or ongoing bleeding, irrespective of ultrasound findings [4,7]. This is based on the understanding that although pelvic ultrasound can aid the exclusion of retained products of conception, the appearance of the immediate postpartum uterus can be unreliable [8,9] no matter which imaging modality is used. It is our belief that a better understanding of postpartum ultrasound findings could enable more accurate identification of women requiring surgical intervention, with

"Corresponding Author: Dr. Aslı Üçyiğit, Early Pregnancy \& Acute Gynaecology Unit, Suite 8, 3rd Floor, Golden Jubilee Wing, King's College Hospital, Denmark Hill, London, SE5 9RS, United Kingdom; E-mail: asli.ucyigit@nhs.net

Citation: Üçyiğit A, Johns J (2017) Imaging of Postpartum Uterine Pathology: A Review. Int J Gynecol Clin Pract 2: 136. https://doi.org/10.15344/23944986/2017/136

Copyright: @ 2017 Üçyiğit, et al. This is an open-access article distributed under the terms of the Creative Commons Attribution License, which permits unrestricted use, distribution, and reproduction in any medium, provided the original author and source are credited. 
Citation: Üçyiğit A, Johns J (2017) Imaging of Postpartum Uterine Pathology: A Review. Int J Gynecol Clin Pract 2: 136. https://doi.org/10.15344/23944986/2017/136

consequent reduction in surgical complications, radiation exposure, and cost to healthcare services.

Occasionally the request for postpartum imaging may follow the clinical finding of a pelvic mass, or suspected adnexal mass accident. It is unlikely that such masses would not have been detected during antenatal screening scans however the discovery of ovarian cysts postpartum is not unheard of. In this setting, should there be diagnostic uncertainty as to the nature of the cyst, an MRI scan may be a useful secondary imaging tool following the initial ultrasound scan.

\section{The Normal Postpartum Uterus}

Immediately following delivery, the action of oxytocin (endogenous or synthetic) onthe uterus causes it to undergo a rapid involution. The average mass of the term uterus post-delivery is $1000 \mathrm{~g}$, at which time the uterine fundus can oftenbe palpated at the level of the umbilicus. By day seven postpartum, the uterine mass has halved, and a further seven days later, the uterine size decreases such that is no longer palpable abdominally, returning to the true pelvis. After six weeks the uterus has decreased to $50-100 \mathrm{~g}$, a size consistent with a non pregnant state, however the final uterine size will remain larger than the original nulligravid state. In contrast to the gradual recovery of the uterus, the postpartum endometrium regenerates at a rapid pace. By the seventh postpartum day, endometrial glands are already formed, and after two weeks the endometrial lining is almost completely restored throughout the uterine cavity, with the exception of the placental bed site [10].

The vast majority of this knowledge was obtained historically from histological analysis of post mortem specimens, when puerperal maternal death rates were higher [11] fortunately, in more recent times, uterine imaging (specifically ultrasound) has been the mainstay in furthering our knowledge of postpartum uterine physiology and appearances.

\section{Radiological Appearances of the Postpartum Uterus}

There is abundant data and description regarding the ultrasound appearances of the process of normal uterine involution in the literature, (Figure 1) [12] however very few studies exist where CT or MRI have been used to image the normal postpartum uterus. Althougha large proportion of these studies span a number of decades, there is a general consensus that a significant overlap between the normal and abnormal uterus exists, across all modes of imaging [9, 12-14].

A more recent study of the postpartum uterus assessed both the anteroposterior (AP) diameter of the uterus and the endometrial thickness (postpartum uterine lining), and the findings indicate that mode of delivery and gestational agecan affectthe rate of uterine involution. For example, the reduction in endometrial thickness over the postpartum course following a term vaginal delivery was found to be significantly greater than that following term caesarean section; additionally, the decrease in the AP diameter of the uterus was less after a preterm delivery versus a term delivery. Although based on a small number of cases, this study supports the theory that the process of uterine involution will vary with both mode and timing of delivery [15].

Despite numerous imaging studies assessing uterine involution, there are conflicting data describing the typical radiological appearances of the uterine cavity and its contents postpartum, and also the relevance of such findings to clinical practice. In order to aid

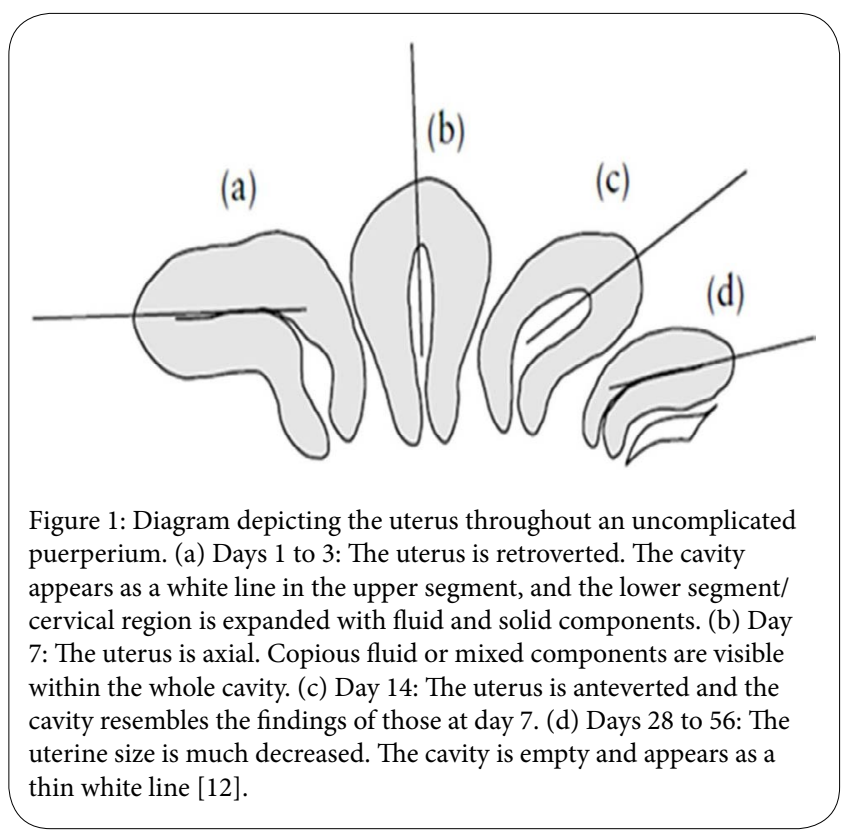

understanding of the physiological and pathological characteristics of the postpartum uterus, radiological assessment of the uterine cavity can be broadly divided into two categories: (1) the immediate post partum period i.e. within the first 24 hours, and (2) 24 hours postdelivery through to the end of the puerperium.

Despite numerous imaging studies assessing uterine involution, there are conflicting data describing the typical radiological appearances of the uterine cavity and its contents postpartum, and also the relevance of such findings to clinical practice. In order to aid understanding of the physiological and pathological characteristics of the postpartum uterus, radiological assessment of the uterine cavity can be broadly divided into two categories: (1) the immediate post partum period i.e. within the first 24 hours, and (2) 24 hours postdelivery through to the end of the puerperium.

\section{Radiological Appearances Immediately Postpartum (within 24 hours)}

The presence of intrauterine blood clots, debris, and/or fluid is common following vaginal delivery, and will appear as areas of increased echogenicity on ultrasonographic assessment. (Figure 2) Should a CT scan be undertaken, a central area of low-attenuation will be revealed, however in the presence of fresh bleeding from the placental bed these clots will appear as hyperattenuating material (Figure 3) [5]. The presence of intracavity gas can be considered a normal postpartum finding in the absence of abnormal symptomatology, and will appear as gas bubbles on CT, or foci of echogenic shadowing on ultrasound.

The volumes of intracavity material have also been studied: in a prospective observational study of 94 women, Deans et al. sought to establish if there was any correlation between transabdominal ultrasound findings and patient morbidity in the first 24 hours postpartum [16]. Unexpectedly large volumes of echogenic material were revealed within the uterine cavity, in particular within the lower segment of the uterus, where mean volumes were as great as $54.8 \mathrm{~cm}^{3}$. However, there appeared to be no correlation between the presence of this material and the development of postpartum morbidity, such as pyrexia, PPH or prolonged hospital stay. This suggests that the presence of large volumes of intrauterine echogenic material in the first day post-delivery can be accepted as normal. 
Citation: Üçyiğit A, Johns J (2017) Imaging of Postpartum Uterine Pathology: A Review. Int J Gynecol Clin Pract 2: 136. https://doi.org/10.15344/2394$4986 / 2017 / 136$

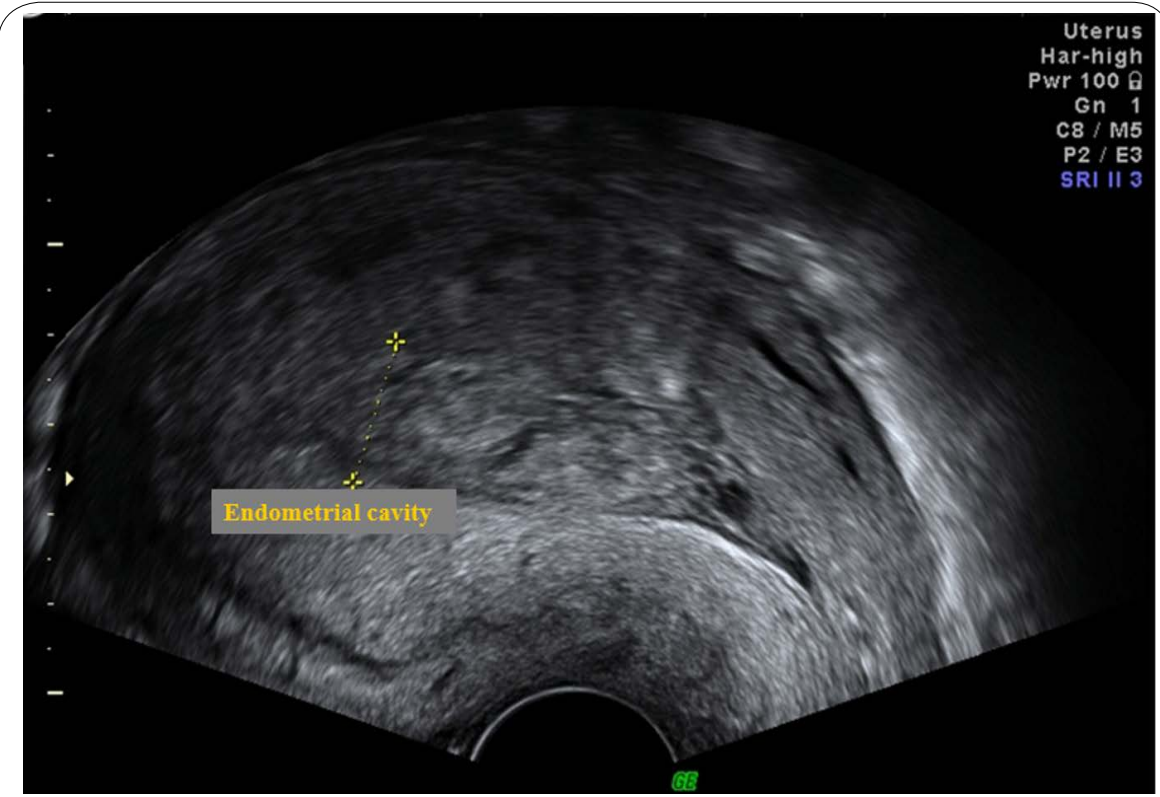

Figure 2: Echogenic debris within the endometrial cavity seen in the sagittal plane on transvaginal scan 12 hours post vaginal delivery.

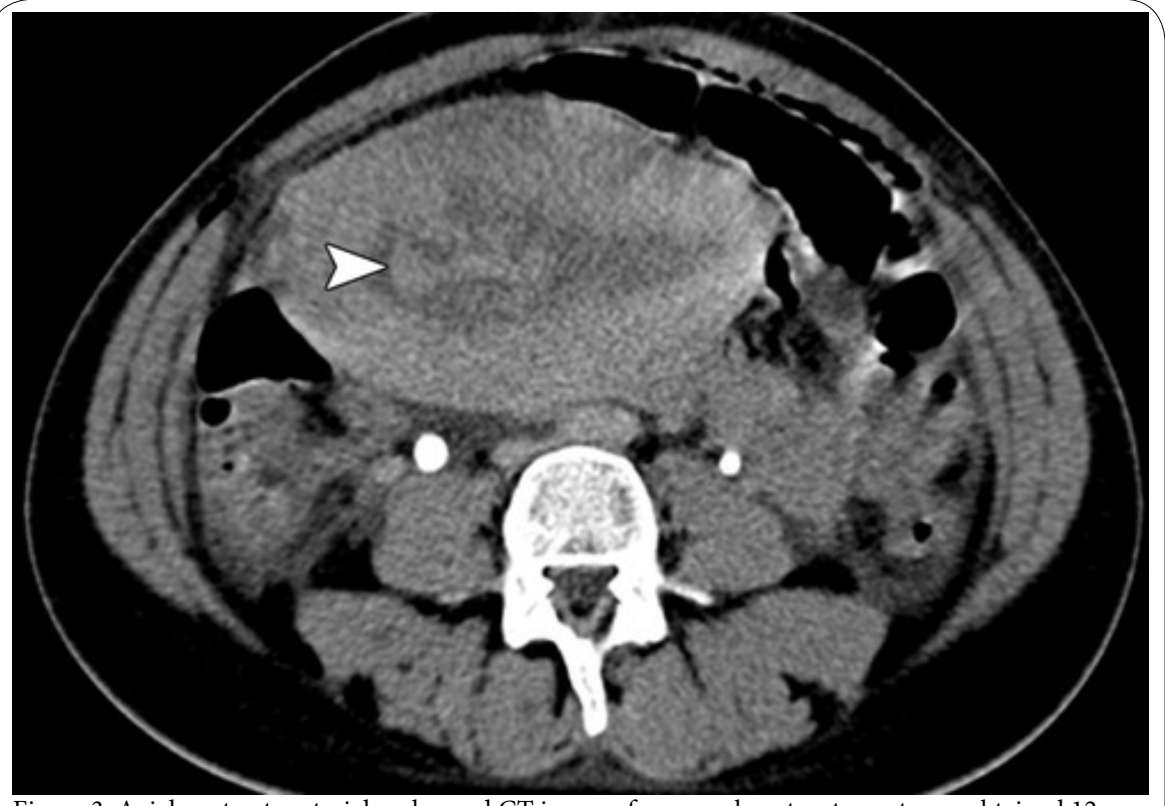

Figure 3: Axial contrast material-enhanced CT image of a normal postpartum uterus, obtained 12 hours after vaginal delivery reveals an enlarged uterus containing a low-attenuation central area corresponding to intrauterine blood debris and fluid. Fresh blood clots are seen as hyperattenuating foci (arrowhead). (Reprinted with permission) [5].

In a similar study, immediate ultrasonographic assessment was undertaken following placental delivery, but with concomitant surgical curettage of the uterine cavity (within two minutes of the scan) [17]. Following histological assessment of the intrauterine material, the sensitivity, specificity, positive and negative predictive value of ultrasound in detecting retained products in their study was $44 \%$, $92 \%, 58 \%$, and $87 \%$, respectively. Of those patients with histologically confirmed retained products of conception, a large proportion in fact had a normal endometrial cavity on ultrasound scan (37.5\%). The remainder had either an echogenic mass, a heterogeneous mixed density mass, or intrauterine fluid alone. The vascularity of these intrauterine masses was however not assessed. Thus it can be concluded that in the absence of colour Doppler assessment, the appearances of retained products immediately following delivery are highly variable and cannot be correlated with a need for intervention.

\section{Radiological Appearances after the first 24 hours Postpartum}

As previously mentioned, the postpartum uterus is often found to contain an accumulation of clinically insignificant debris and fluid, mainly in the lower segment initially and then within the whole uterine cavity by the middle of the puerperium [12]. Various studies have also suggested that the presence of intracavity gas, visible on imaging for several weeks postpartum (Figure 4), can also be accepted as a normal puerperal finding $[18,19]$.

In a recent observational study a small group of women were systematically scanned at weekly intervals postpartum, starting from week one up until week three [9]. These ultrasound assessments revealed that in women with normal postpartum bleeding, there was an echogenic mass in $51 \%$ on day seven, in $21 \%$ on day 14 and in 
Citation: Üçyiğit A, Johns J (2017) Imaging of Postpartum Uterine Pathology: A Review. Int J Gynecol Clin Pract 2: 136. https://doi.org/10.15344/23944986/2017/136

$6 \%$ on day 21 . They found no difference in either the heaviness or bleeding duration between women with and without an echogenic mass at each of these three scans. Thus, the authors hypothesised that either an echogenic mass does not always represent retained products of conception, or that products of conception are commonly retained and are therefore of little clinical significance in many cases. However, Doppler assessment of these products did not appear to be undertaken, nor was there any clarification by the authors as to whether the description of an echogenic mass also included mixedecho patterns, a finding which other studies have suggested is an insignificant postpartum occurrence [20]. Care must therefore be taken to interpret ultrasound scan findings in this clinical context; the specific finding of an echogenic mass in the setting of secondary PPH is likely to be associated with retained placental tissue and requires surgical intervention, whereas mixed-echo patterns may not, and can be managed expectantly, with early resolution of symptoms [20].

The addition of colour Doppler assessment at ultrasound scan can aid identification of placental remnants [21]. A cross-sectional study of 385 postnatal women revealed areas of enhanced vascularity in 32 women $(8.3 \%)$, and 26 women $(6.75 \%)$ had retained placental products on scan. Although no comment was made regarding patient morbidity, a high incidence of histological confirmation was obtained following surgical curettage (19 of 20 cases), suggesting that the use of colour Doppler may be of practical diagnostic value [21]. The following images demonstrate the use of colour Doppler in aiding the diagnosis of retained products of conception, later confirmed on histological analysis.

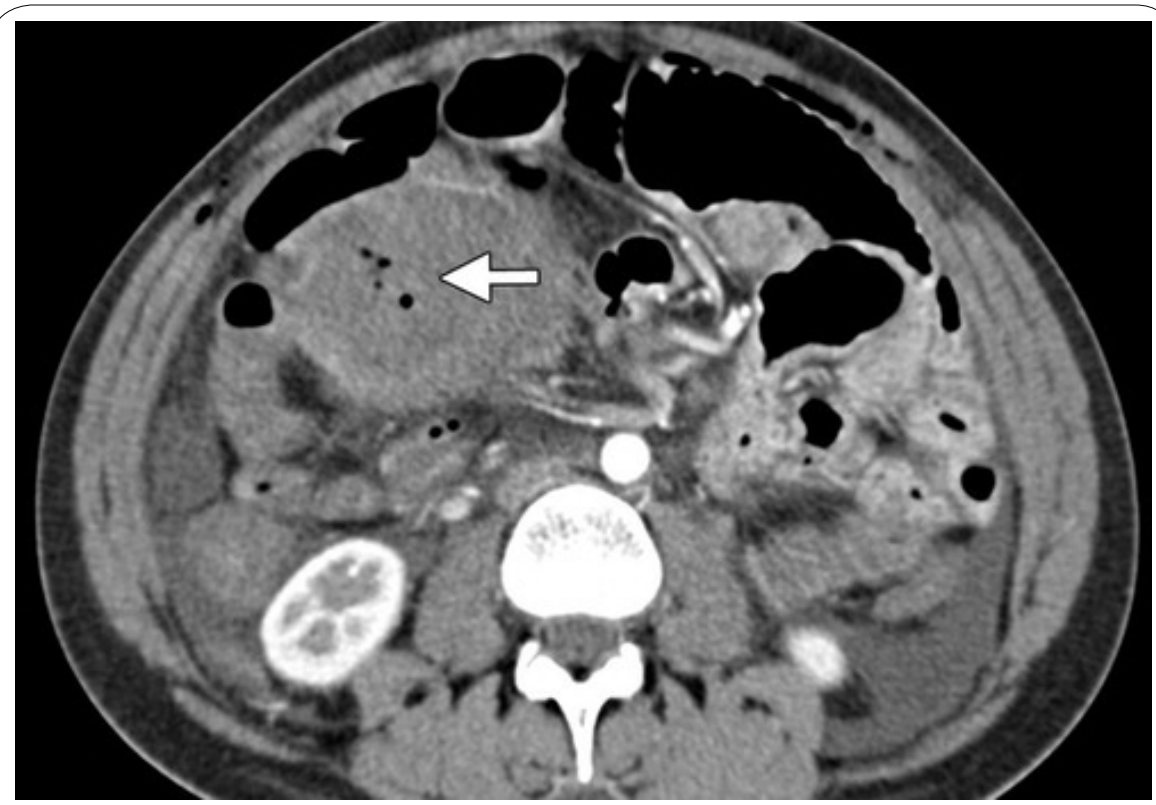

Figure 4: Axial contrast-enhanced CT image shows intrauterine gas bubbles (arrow), which are a normal postpartum finding in the absence of other symptoms.(Reprinted with permission) [5].

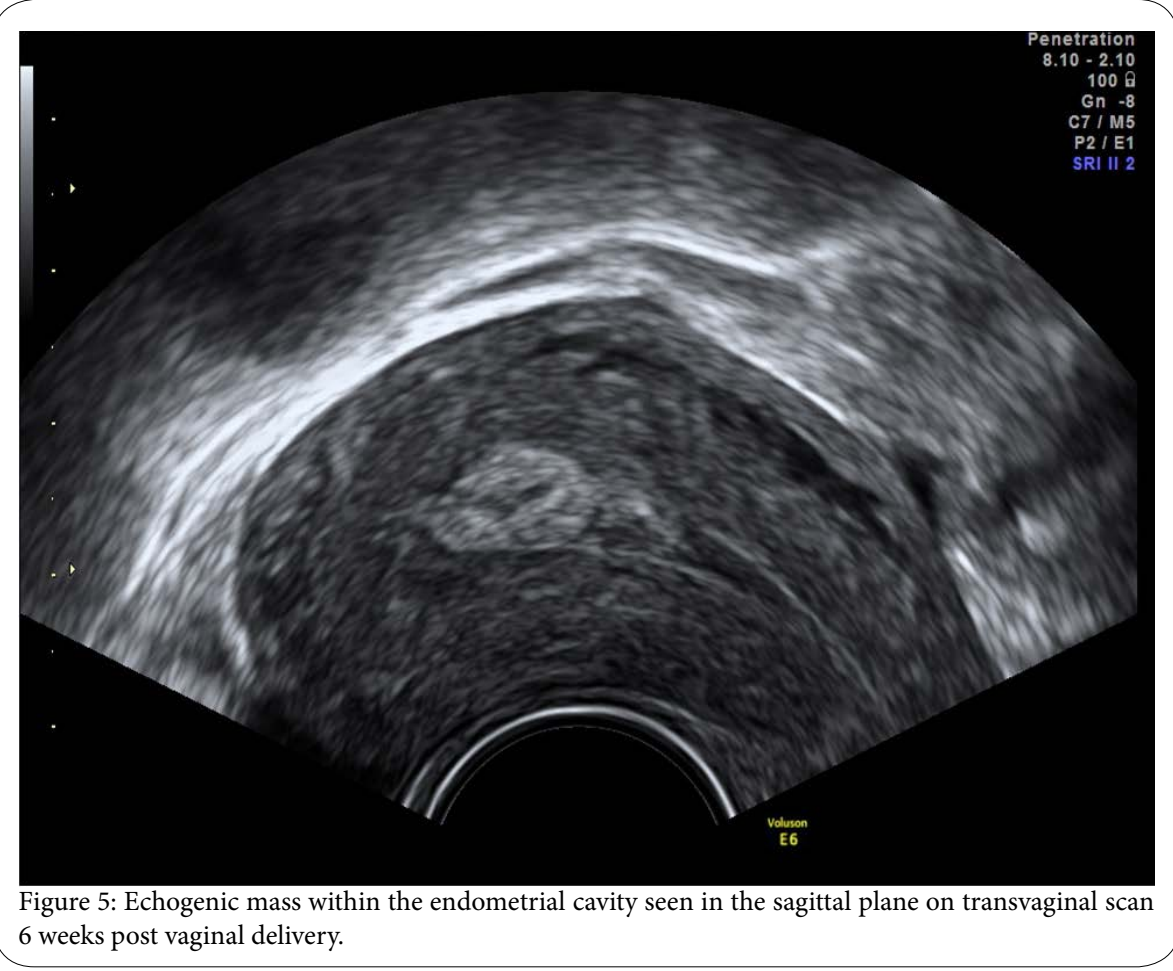


Citation: Üçyiğit A, Johns J (2017) Imaging of Postpartum Uterine Pathology: A Review. Int J Gynecol Clin Pract 2: 136. https://doi.org/10.15344/2394$4986 / 2017 / 136$

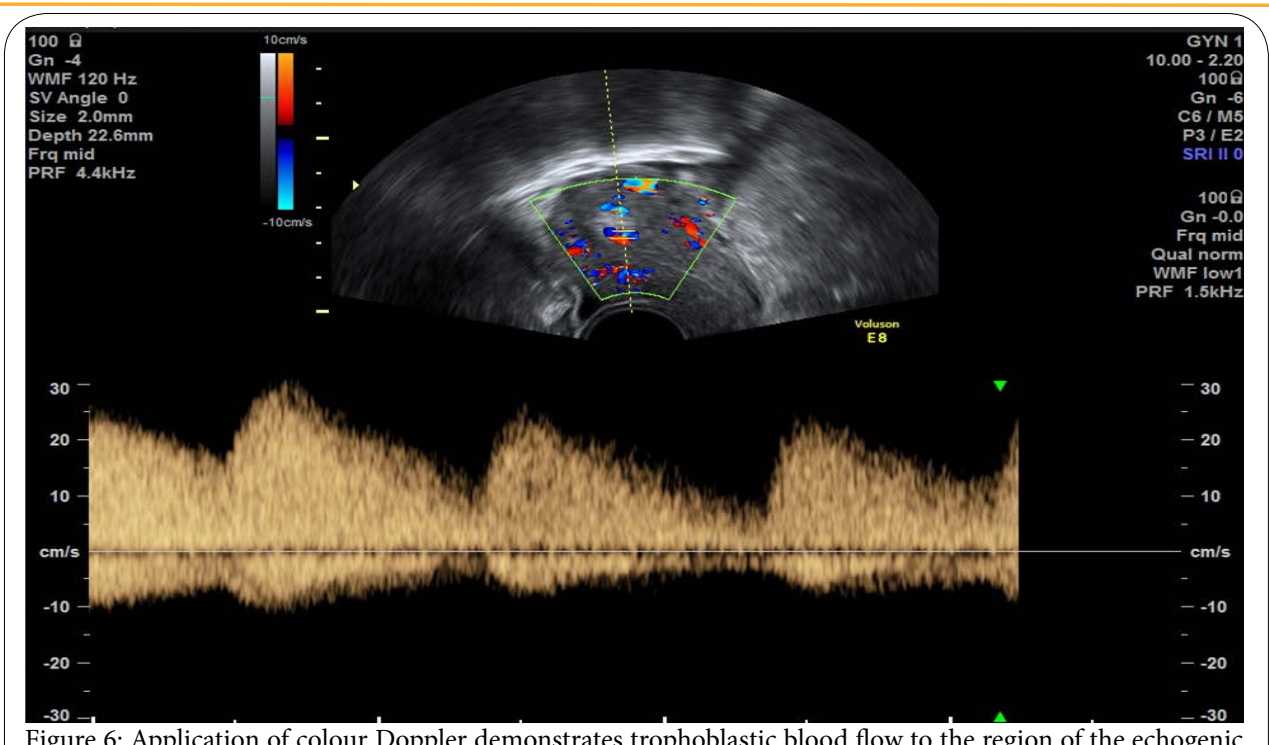

Figure 6: Application of colour Doppler demonstrates trophoblastic blood flow to the region of the echogenic mass, aiding diagnosis of retained products of conception.

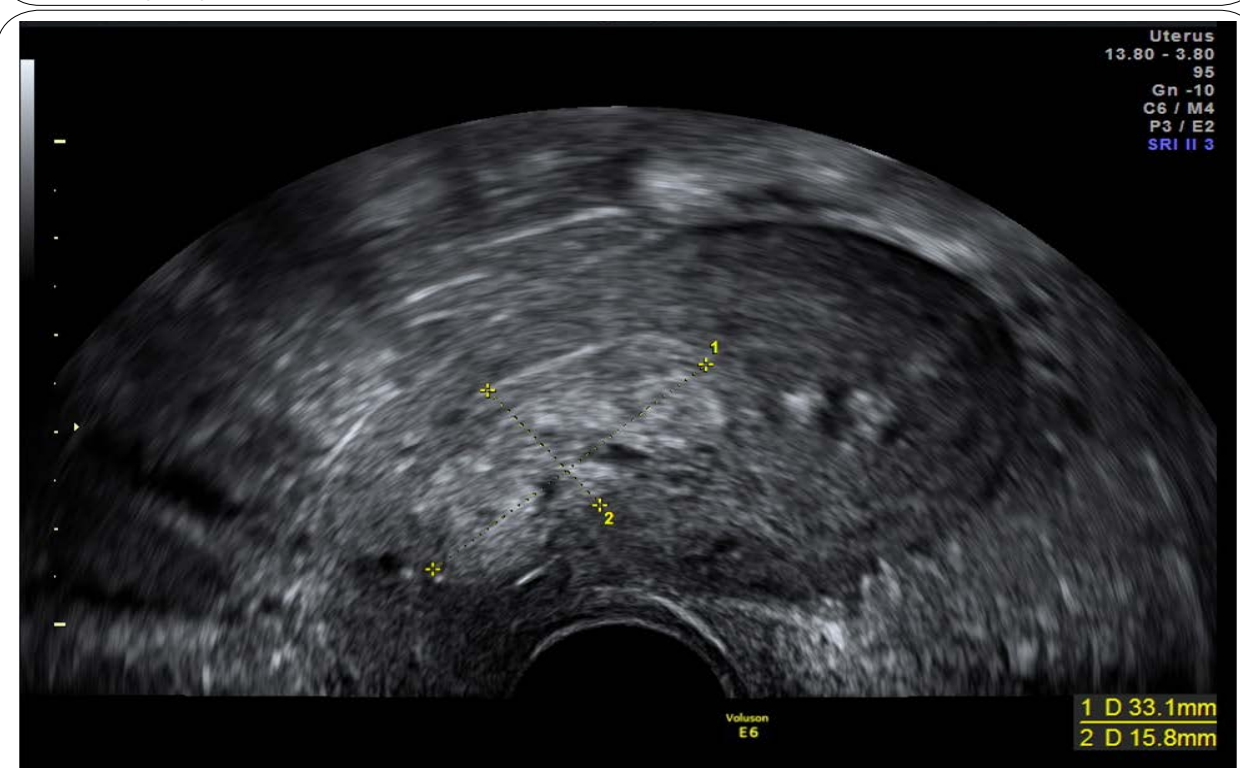

Figure 7: Echogenic mass seen within the lower uterine segment in the sagittal plane on transvaginal scan performed in a symptomatic patient 8 weeks post vaginal delivery.

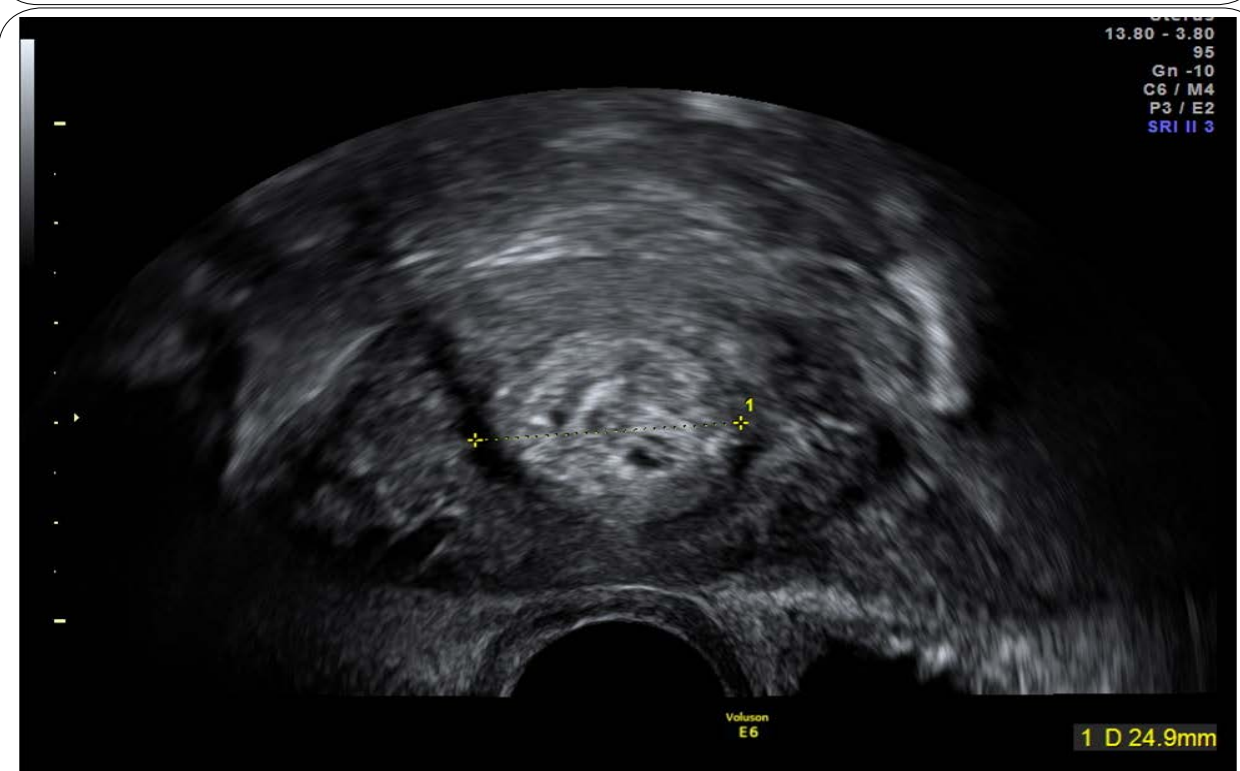

Figure 8: Echogenic mass within the lower uterine segment in the same patient (Figure 7) as seen in the transverse plane on transvaginal scan. 
Citation: Üçyiğit A, Johns J (2017) Imaging of Postpartum Uterine Pathology: A Review. Int J Gynecol Clin Pract 2: 136. https://doi.org/10.15344/23944986/2017/136

\section{Rare Postpartum Pathology and Imaging Findings}

Less common postpartum ultrasound findings are summarised below.

\section{Caesarean Section Associated Findings}

Globally rising rates of caesarean section delivery have led to an increased understanding of normal and abnormal post-caesarean imaging findings. Following an uncomplicated procedure, ultrasound will identify the uterine incision as an iso- or hypoechoic region when compared to myometrium, and when imaged in the sagittal plane on transvaginal scan, is centrally located between the uterus and bladder [22-24]. Depending on probe orientation, the uterine sutures can be identified as linear or point-like hyperechoic foci, and small haematomas $(<15 \mathrm{~mm})$ along the suture line can be considered as normal [22-24]. On CT, the incision site will demonstrate transverse focal thinning; as the transverse incision will be parallel to the axial imaging plane, sagittal reformatted CT images are better in demonstrating the area of decreased attenuation that will be present in the lower uterine segment (Figure 10) [5]. The images can be further enhanced through intravenous contrast administration, however resulting image quality will always be limited due to the relative fluid overload associated with pregnancy and surgery [22].

If adequate haemostasis has not been achieved intraoperatively, the immediate post-operative period may be complicated by the formation of a bladder flap haematoma. During a lower segment caesarean section, the visceral peritoneum is incised between the uterus and bladder, and reflected inferiorly. It is in this space that a haematoma may form, and on ultrasound will be seen as a non-vascular mass of mixed echogenicity, anterior to the uterus and posterior to the bladder, and has been described as a 'bladder flap' haematoma in the literature [5,22]. (Figures $11 \& 12$ ) These may or may not be contained by the overlying peritoneum, and in the latter scenario will lead to the detection of haematoperitoneum on scan. At CT, the bladder flap haematoma will appear as a slightly hyperattenuating area in the same location (Figure 13), with possible mass effect [5,22]. The presence of such a haematoma is generally considered to be of little clinical significance if they are below $4 \mathrm{~cm}$ in size [22].

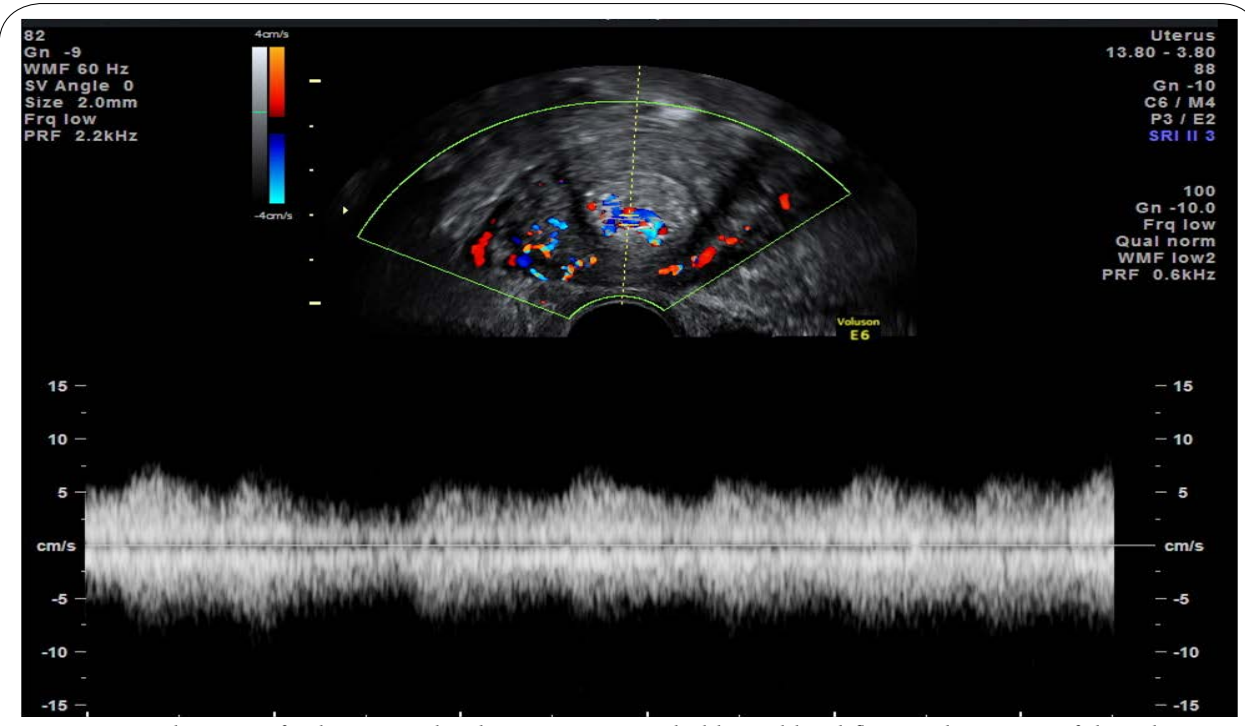

Figure 9: Application of colour Doppler demonstrates trophoblastic blood flow to the region of the echogenic mass in the transverse plane on transvaginal scan (as seen in Figure 8) aiding diagnosis of retained products of conception.

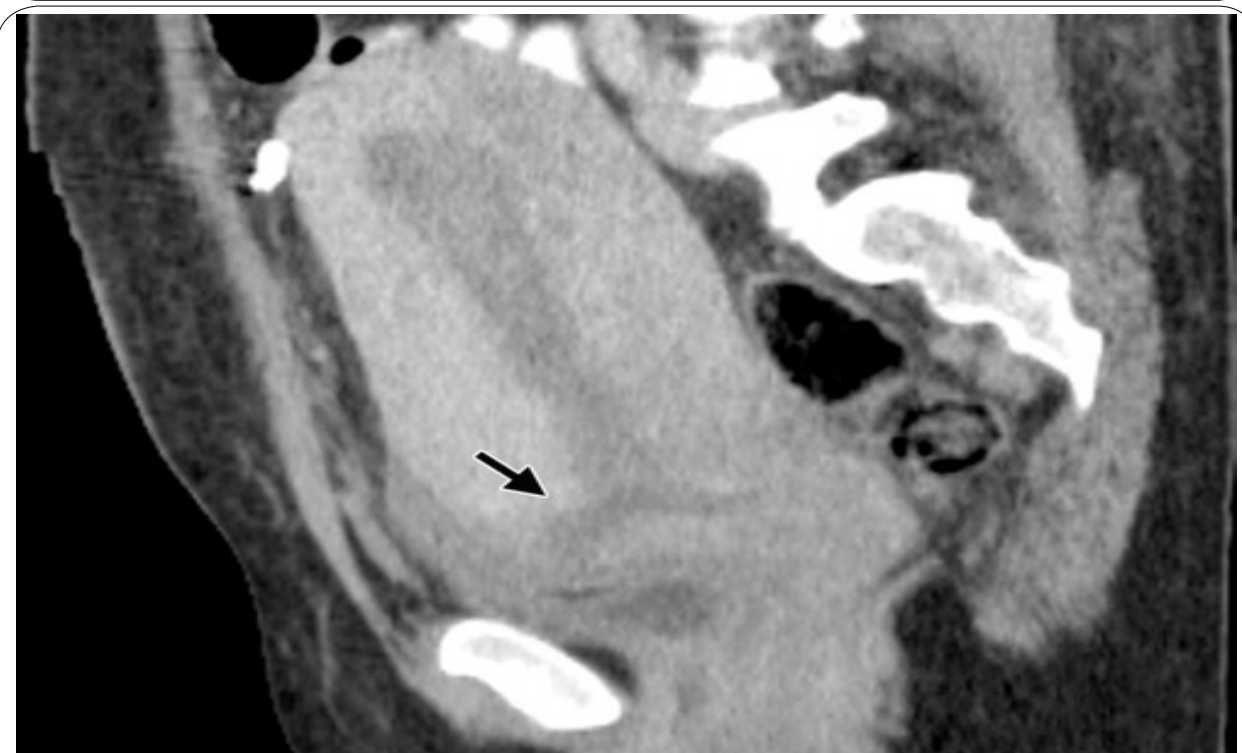

Figure 10: Sagittal portal phase reformatted CT image following a normal caesarean section reveals a low transverse discontinuity (arrow) at the incision site in the anterior portion of the lower uterine segment. (Reprinted with permission) [5]. 
Citation: Üçyiğit A, Johns J (2017) Imaging of Postpartum Uterine Pathology: A Review. Int J Gynecol Clin Pract 2: 136. https://doi.org/10.15344/2394$4986 / 2017 / 136$
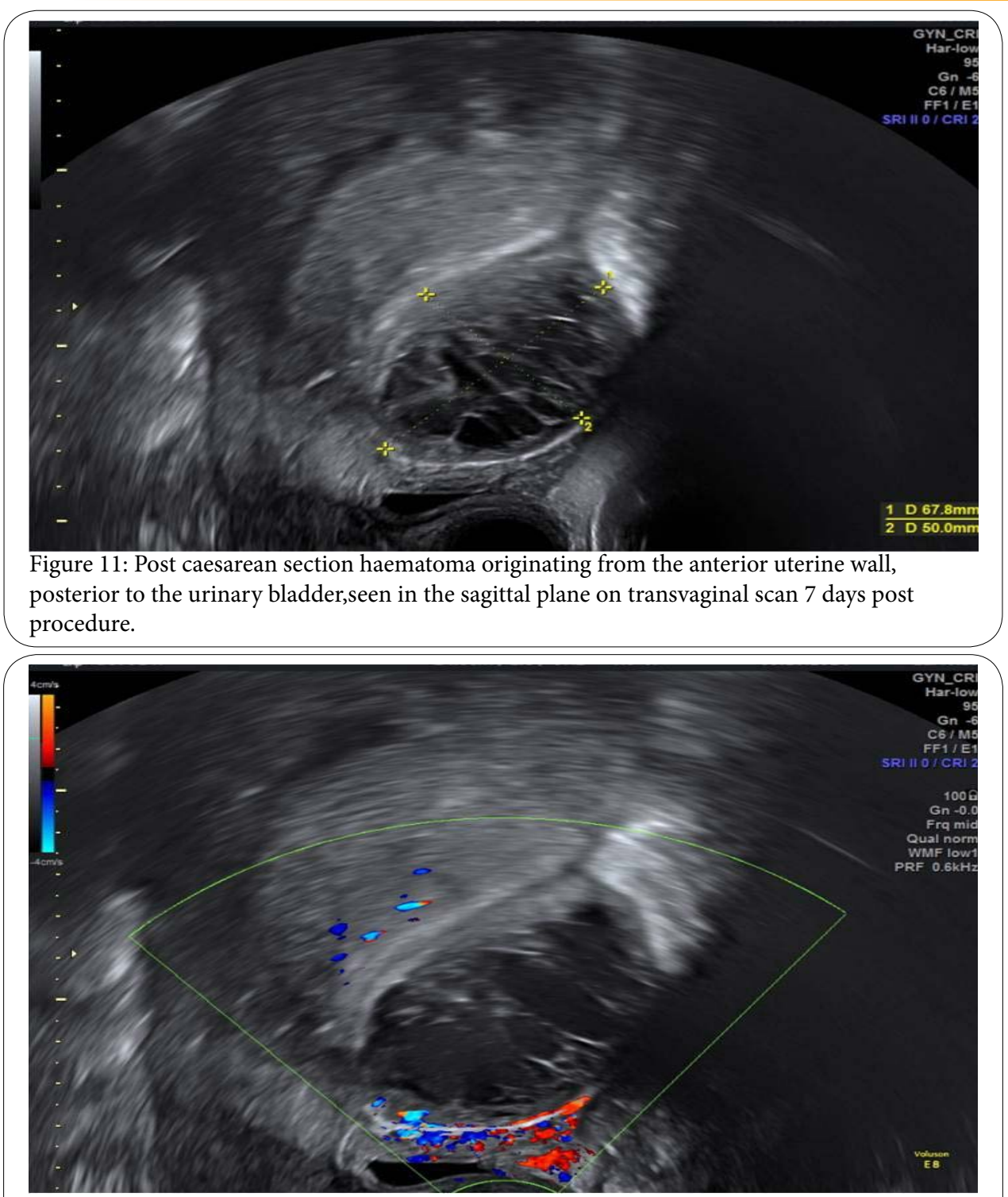

Figure 12: Colour Doppler shows a non-vascular mixed echogenicity haematomaoriginating from the anterior uterine wall, posterior to the urinary bladder (sagittal plane on transvaginal scan).

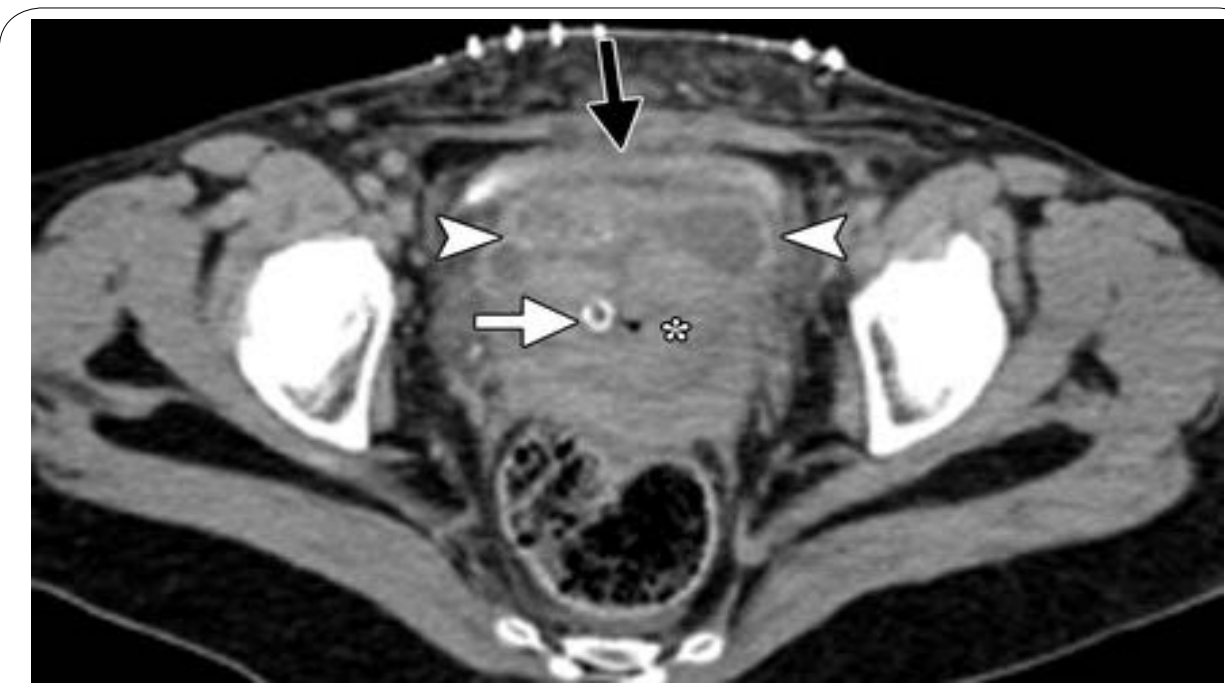

Figure 13: Axial contrast-enhanced CT image reveals a fluid collection (arrowheads) anterior to the lower uterine segment $\left({ }^{*}\right)$ and posterior to the bladder (black arrow), corresponding to a small bladder flap hematoma post caesarean section. The intrauterine balloon catheter can be seen (white arrow) [5]. 
Citation: Üçyiğit A, Johns J (2017) Imaging of Postpartum Uterine Pathology: A Review. Int J Gynecol Clin Pract 2: 136. https://doi.org/10.15344/23944986/2017/136

\section{Pelvic sepsis}

Few studies exist which specifically report the imaging findings expected in postpartum endometritis, however in contrast, cases of pelvic sepsis with underlying abscesses are frequently described. $[22,25,26]$ Superimposed infection of a pelvic or bladder flap haematoma may lead to the formation of a pelvic abscess, the diagnosis of which can be achieved with ultrasound. The abscess will appear as a well-circumscribed fluid collection with or without internal septations, containing internal debris. The presence of gas, seen if gas-producing organisms are present, will cause multiple highly echogenic foci, leading to dirty posterior shadowing on scan [22]. CT scan will reveal a gas containing fluid collection, again with or without internal septations, and will be rim-enhancing [22].

\section{Arteriovenous malformations}

With now over 200 cases reported in the literature, uterine arteriovenous malformations (AVMs) are not as rare as once thought, following the first reports almost 90 years ago [27]. These pelvic lesions are an acquired anomaly and an association with trophoblastic disease, pelvic surgery (e.g. myomectomy), endometrial curettage, uterine malignancy and caesarean scar pregnancy have all been reported. A congenital aetiology has been postulated, particularly in cases where there is multiorgan involvement and the presence of multiple AVMs. They are most prevalent in women of reproductive age, rarely occurring in the nulligravid. Thus it has been hypothesised that pregnancy contributes to the pathogenesis of uterine AVMs [28] where necrosis of chorionic villi leads to the incorporation of venous sinuses into areas of myometrial scarring.

The clinical presentation may be with either primary or secondary $\mathrm{PPH}$, and rarely, a pulsatile pelvic mass. The volume of blood loss can be extensive and swift, leading to rapid haemodynamic compromise. Although the current gold standard diagnostic test is pelvic angiography, the use of Doppler ultrasound can successfully identify these vascular lesions. The typical appearance is of a highly vascular localised area within the myometrium. (Figure 14) Pulsed Doppler evaluation will usually reveal a low-resistance blood flow with a broad waveform, high peak velocities and signs of turbulence. [28] Treatment is typically with selective embolisation of the feeding vessel, and less often surgical excision of the lesion. Both CT and MRI can be useful in the identification of feeding vessels; contrast CT will reveal the numerous tortuous vessels coursing through the parametrium and

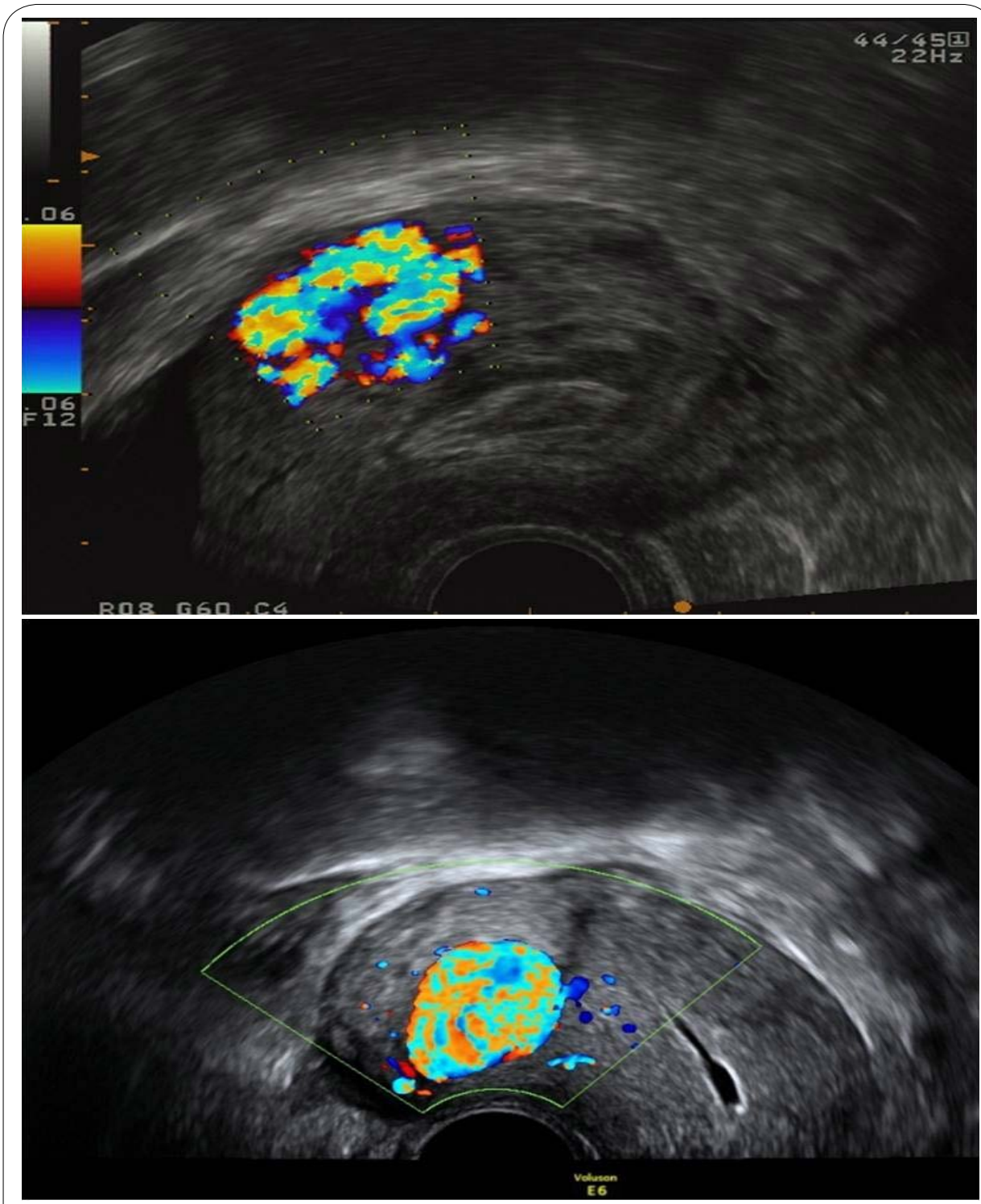

Figures 14: Colour Doppler reveals localised, highly vascular AVMs within the myometrium identified on transvaginal scan (sagittal plane). 
Citation: Üçyiğit A, Johns J (2017) Imaging of Postpartum Uterine Pathology: A Review. Int J Gynecol Clin Pract 2: 136. https://doi.org/10.15344/2394$4986 / 2017 / 136$

uterine wall, and MRI will demonstrate a localised disruption of the uterine junctional zone with multiple serpiginous signal voids [26].

\section{Uterine dehiscence and rupture}

An incomplete rupture of the uterine wall, where by the serosal layer of the uterus remains intact is classed as uterine dehiscence. Should this layer also be disrupted then the more serious complication of caesarean delivery, uterine rupture, has occurred. Uterine dehiscence poses a diagnostic challenge due to the significant overlap with the normal uterine appearances post caesarean section. The presence of a bladder-flap haematoma greater than $5 \mathrm{~cm}$ in size identified on either ultrasound or CT should raise the suspicion for uterine dehiscence [22]. In this clinical setting, an MRI scan may be superior for confirming the diagnosis in view of its enhanced soft-tissue contrast and multiplanar capability, enabling depiction of an intact serosal layer [22]. In contrast, the diagnosis of uterine rupture can be more readily achieved on ultrasound or CT scan. Focal disruption of the myometrium, the presence of gas extending from the endometrial cavity to the parametrium, and the addition of haematoperitoneum are imaging findings that will typically provide the diagnosis (Figure $15 a$ and Figure 15b) $[5,22]$.

\section{Conclusions}

Ultrasound can be considered the first-line imaging modality of choice in the assessment of puerperal uterine pathology, providing a safer and more acceptable experience for the patient, and an accessible and relatively inexpensive option for the clinician. In the event of rarer postpartum complications with consequent diagnostic uncertainty, $\mathrm{CT}$ is often used as a secondary imaging adjunct. However in order to obtain more detailed assessment of the uterine soft tissues (e.g. should dehiscence be suspected) MRI is the modality of choice. It is, therefore, key that the most appropriate imaging modality is chosen for the accurate assessment and characterisation of postpartum uterine pathology, thus ensuring timely and correct treatment of the puerperal patient.

\section{Competing Interests}

The author(s) declared no potential conflicts of interest with respect to the research, authorship, and/or publication of this article.
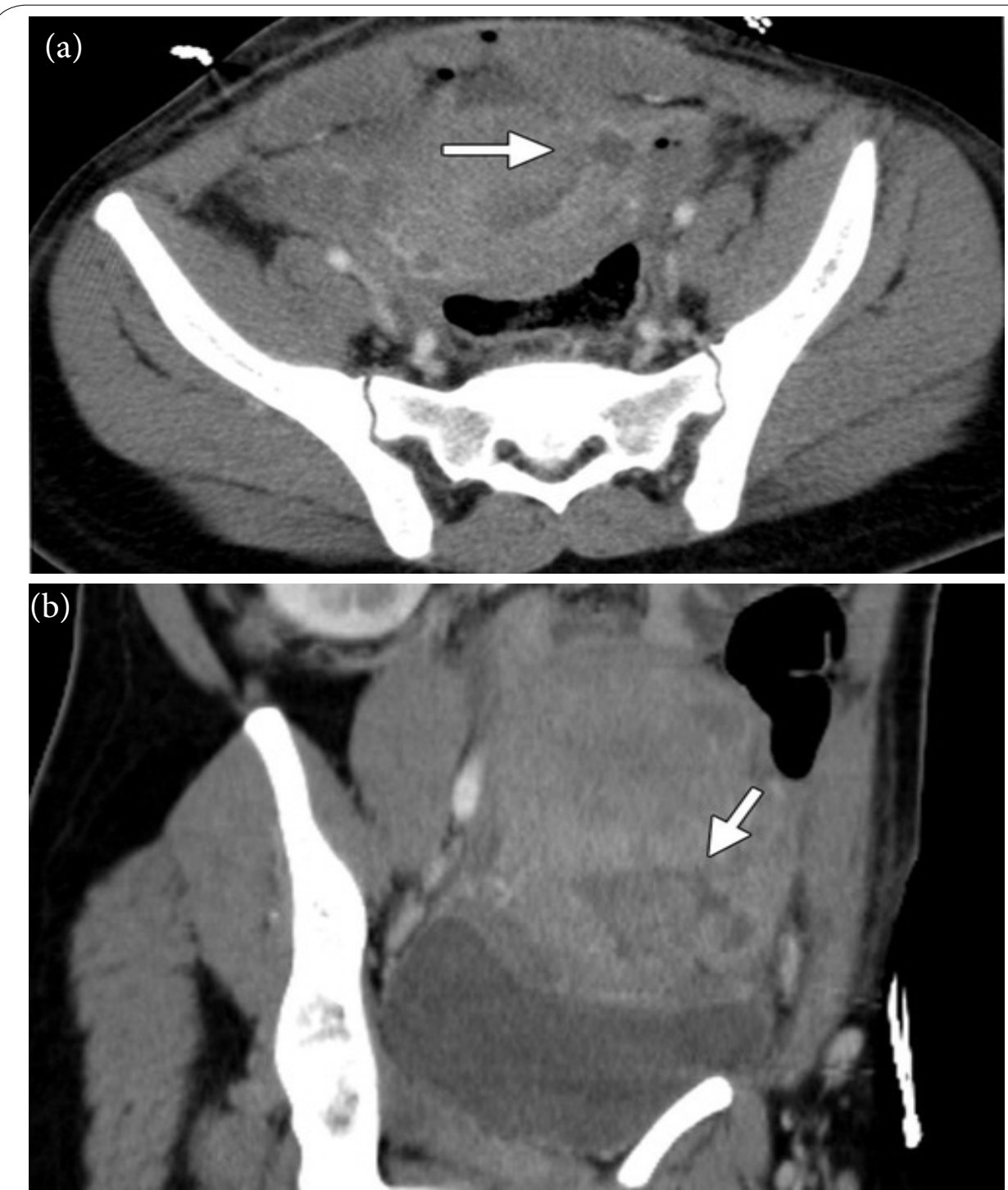

Figures 15 : Contrast-enhance CT images taken in the axial (a) and coronal oblique (b) planes, 4 days post caesarean delivery. Disruption of the myometrium (arrow) in the left aspect of the lower uterine segment can be seen as a hypoattenuating band in the myometrium, with subsequent communication of the uterine cavity with a fluid collection in the left broad ligament and pelvis (Reprinted with permission) [5]. 
Citation: Üçyiğit A, Johns J (2017) Imaging of Postpartum Uterine Pathology: A Review. Int J Gynecol Clin Pract 2: 136. https://doi.org/10.15344/23944986/2017/138

\section{References}

1. Royston E, Armstrong S, WHO (1989) Preventing Maternal Deaths. Geneva: World Health Organization.

2. World Health Organization (1990) The Prevention and Management of Postpartum Haemorrhage. Report of a Technical Working Group. Geneva: WHO.

3. Mousa HA, Blum J, Abou El Senoun G, Shakur H, Alfirevic Z, et al. (2007) Treatment for primary postpartum haemorrhage. Cochrane Database Syst Rev 13: CD003249.

4. Royal College of Obstetrics \& Gynaecology (2009) Green top Guideline No. 52: Postpartum Haemorrhage, Prevention and Management.

5. Sierra A, Burrel M, Sebastia C, Radoseviv A, Barrufet M, et al. (2012) Utility of multidetector CT in severe postpartum haemorrhage. Radiographics 32 : 1463-1481.

6. Alexander J,Thomas PW, Sanghera J (2002) Treatments for secondary postpartum haemorrhage. Cochrane Database Syst Rev.

7. Collins S, Arulkumaran S, Hayes K, Jackson S, Impey L, et al. (2013) Oxford Handbook of Obstetrics \& Gynaecology. Third Edition. Oxford: Oxford University Press. pp 323-323.

8. Sadan O, Golan A, Girtler O, Lurie S, Debby A, et al. (2004) Role of sonography in the diagnosis of retained products of conception. J Ultrasound Med 23: 371-374.

9. Edwards A, Ellwood DA (2000) Ultrasonographic evaluation of the postpartum uterus. Ultrasound Obstet Gynecol 16: 640-643.

10. Spiliopoulos M, Mastrogiannis D (2013) Normal and Abnormal Puerperium.

11. Hytten F (1995) The Clinical Physiology of the Puerperium. London: Farrand Press 65: 259

12. Mulic-Lutvica A, Bekuretsion M, Bakos O, Axelsson O (2001) Ultrasonic evaluation of the uterus and uterine cavity after normal, vaginal delivery. Ultrasound Obstet Gynecol 18: 491-498

13. Wachsberg RH, Kurtz AB, Levine CD, Solomon P, Wapner RJ, et al. (1994) Real-time ultrasonographic analysis of the normal postpartum uterus: technique, variability, and measurements. J Ultrasound Med 13: 215-221.

14. Lavery JP, Shaw LA (1989) Sonography of the puerperal uterus. J Ultrasound Med 8: 481-486

15. Bae HS, Ahn KH, Oh MJ, Kim HJ, Hong SC, et al. (2012) Postpartum uterine involution: sonographic changes in the endometrium between 2 and 6 weeks postpartum related to delivery mode and gestational age at delivery. Ultrasound Obstet Gynecol 39: 727-728.

16. Deans R, Dietz HP (2006) Ultrasound of the post-partum uterus. Aust N Z J ObstetGynaecol 46: 345-349.

17. Carlan SJ, Scott WT, Pollack R, Harris K (1997) Appearance of the uterus by ultrasound immediately after placental delivery with pathologic correlation. J Clin Ultrasound 25: 301-308.

18. Kamaya A, Ro K, Benedetti NJ, Chang PL, Desser TS, et al. (2009) Imaging and diagnosis of postpartum complications: sonography and other imaging modalities. Ultrasound Q 25: 151-162.

19. Brown DL (2005) Pelvic ultrasound in the post abortion and postpartum patient. Ultrasound Q 21: 27-37.

20. Mulic-Lutvica A, Axelsson $O$ (2006) Ultrasound finding of an echogenic mass in women with secondary postpartum haemorrhage is associated with retained placental tissue. Ultrasound Obstet Gynecol 28: 312-319.

21. Van den Bosch T, Van Schoubroeck D, Lu C, De Brabanter J, Van Huffel $S$, et al. (2002) Color Doppler and gray-scale ultrasound evaluation of the postpartum uterus. Ultrasound Obstet Gynecol 20: 586-591.

22. Rodgers SK, Kirby CL, Smith RJ, Horrow MM (2012) Imaging after cesarean delivery: acute and chronic complications. Radiographics 32: 1693-1712.

23. Baker ME, Kay H, Mahony BS, Cooper CJ, Bowie JD, et al. (1998) Sonography of the low transverse incision, cesarean section: a prospective study. J Ultrasound Med 7: 389-393.

24. Koutsougeras G, Karamanidis D, Chimonis G, Gottas N, Polydorou A, et al. (2003) Evaluation during early puerperium of the low transverse incision after cesarean section through vaginal ultrasonography. Clinical and Experimental Obstetrics \& Gynecology 30: 245-247.

25. Leyendecker JR, Gorengaut V, Brown JJ (2004) MR imaging of maternal diseases of the abdomen and pelvis during pregnancy and the immediate postpartum period. Radiographics 24: 1301-1316.

26. Laifer-Narin SL, Kwak E, Kim H, Hecht EM, Newhouse JH, et al. (2014) Multimodality imaging of the postpartum or posttermination uterus: evaluation using ultrasound, computed tomography, and magnetic resonance imaging. Curr Probl Diagn Radiol 43: 374-385.
27. Dubreuil G, Loubat E (1926) Aneurisme circoid de l'uteerus. Ann Anat Pathol 3: 697-718.

28. Kelly SM, Belli AM, Campbell S (2003) Arteriovenous malformation of the uterus associated with secondary postpartum hemorrhage. Ultrasound ObstetGynecol 21: 602-605. 